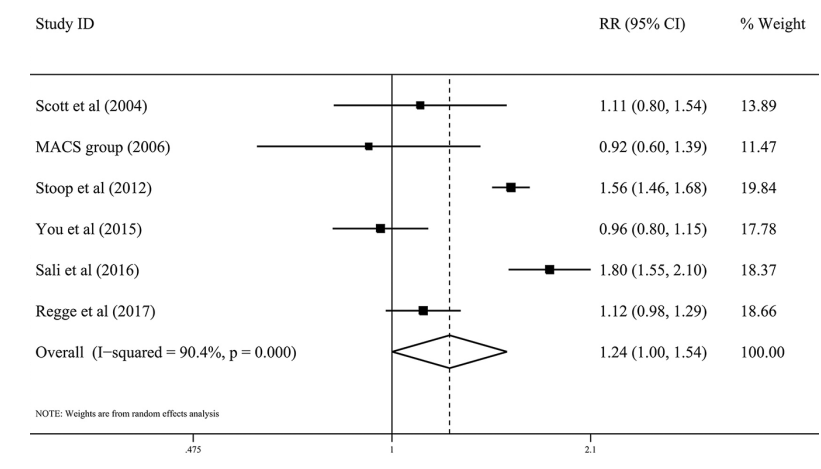

Abstract IDDF2019-ABS-0178 Figure 2

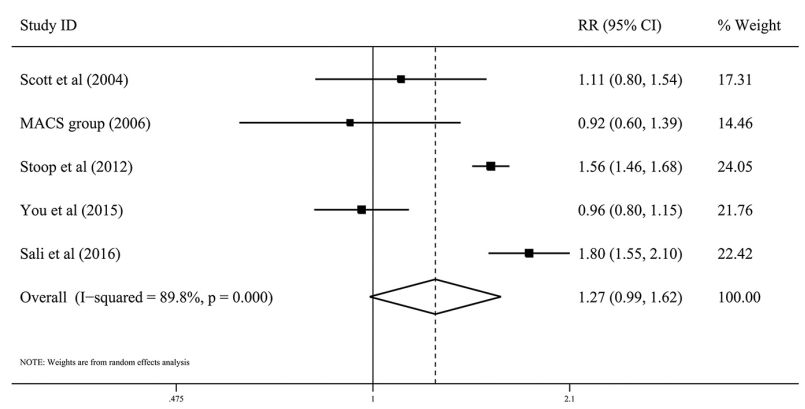

Abstract IDDF2019-ABS-0178 Figure 3

Background To compare the participation rate between CT colonography and colonoscopy in screening population among randomized controlled trials (RCTs).

Methods A search was performed using the PubMed, Web of Science, and Cochrane databases. Studies including unselected screening populations reporting participation rates were limited to randomized controlled trials published in English. The quality of the included articles was ranked in accordance with the Jadad composite scale. Stata 12.0 was used to analyze the data. Risk ratios (RR) were used to compare trials and assess the value of the screening method used. The Higgins $\mathrm{I}^{2}$ statistic was used to determine the total variation across studies due to heterogeneity. If the $\mathrm{I}^{2}$ statistic was $\leq 50 \%$, the findings among the studies were considered homogeneous, and the fixed effects model was used to pool studies; otherwise, the random-effects model was used.

Results Six of 760 studies were included in this meta-analysis (figure 1). There were 17958 invitees in total. Participation rates were $29.0 \%(2716 / 9378)$ for CT colonography and $21.5 \%(1841 / 8580)$ for colonoscopy, and the difference was statistically significant ( $R R=1.241 ; 95 \%$ CI:1.001 to 1.538; $\mathrm{P}=0.049 ; \mathrm{I}^{2}=90.4 \%$, (figure 1)).

We excluded one study used flexible sigmoidoscopy (FS), but the participation rate was not with statistically significantly different between the two groups (pooled $\mathrm{RR}=1.266$; $95 \%$ CI: 0.988 to $1.623 ; \mathrm{P}=0.063 ; \mathrm{I}^{2}=89.8 \%$, (figure 3 )). However, when the two low-quality studies which also were communitybased studies excluded again in these six studies, we found that the participation rate in the CT colonography group was significantly higher than that in the colonoscopy group (pooled $\mathrm{RR}=1.327 ; 95 \% \mathrm{CI}: 1.034$ to $1.702 ; \mathrm{P}=0.026$; $\left.\mathrm{I}^{2}=93.4 \%\right)$.

Conclusions The participation rate in colorectal cancer screening with CT colonography was significantly higher than with colonoscopy. This rate also has a trend over time and sample size. In the future, more large-sample RCTs are needed to confirm the reliability of this study.

\section{IDDF2019-ABS-0180 ASCITES IN ACUTE PANCREATITIS: NOT A SILENT BYSTANDER}

${ }^{1}$ Roshan Agarwala*, 'Jayanta Samanta, ${ }^{1}$ Atul Rana, ${ }^{1}$ Narendra Dhaka, ${ }^{1}$ Pankaj Gupta, ${ }^{1}$ Saroj Kant Sinha, ${ }^{2}$ Vikash Gupta, ${ }^{2}$ Thakur Deen Yadav, ${ }^{1}$ Rakesh Kochhar. ${ }^{1}$ Department of Gastroenterology, Post Graduate Institute of Medical Education and Research, Chandigarh, India; ${ }^{2}$ Department of Surgery, Post Graduate Institute of Medical Education and Research, Chandigarh, India

\subsection{6/gutjnl-2019-IDDFabstracts. 185}

Background Ascites in patients of acute pancreatitis (AP) is understudied although recent literature hints at its evident role on the final outcome. This study was planned to study the characteristics of ascites in patients of AP and its effect on the disease course and outcome.

Methods Consecutive patients of AP were included and patients with or without ascites were evaluated for the baseline parameters and severity assessment. Ascites was quantified and fluid analyzed for its characteristics. Intraabdominal pressure (IAP) was monitored. The various outcome parameters were compared between the two groups of patients with and without ascites.

Results Of the cohort of 213 patients, 82 (38.5\%) developed ascites. Ascites group had significantly higher rates of organ failure $(\mathrm{p}=0.001)$, necrosis $(\mathrm{p}=<0.001)$ and higher severity assessment scores. The ascites group had significantly longer hospital and ICU stay and higher ventilator days compared to the non-ascites group. Mortality was higher in the ascites group $(34.1 \%$ vs $8.45 ; \mathrm{p}=0.001)$. In the ascites group, the majority had moderate to gross ascites (75.6\%), low serumascites albumin gradient (87.8\%) with low amylase levels (71.9\%). Sub-group analysis in ascites group showed that

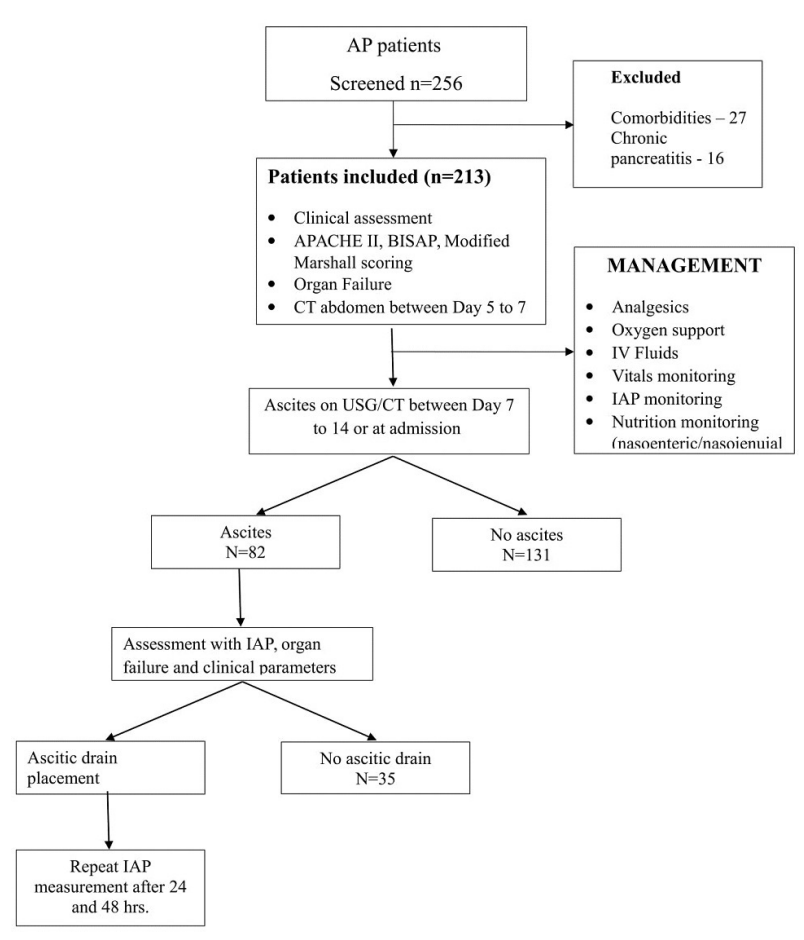

Abstract IDDF2019-ABS-0180 Figure 1 Study design 
patients with fatal outcome had higher rates of moderate to gross ascites, baseline IAP and lower reduction in IAP after 48 hrs. There was no difference in mortality between patients with high $(>1000 \mathrm{U} / \mathrm{L})$ and low $(<1000 \mathrm{U} / \mathrm{L})$ amylase values. On multiple logistic regression analysis, moderate to gross ascites (OR-5.84, CI- 1.08 to 32.01) and grades of intraabdominal hypertension [Grade 1 vs grade 0 (OR-5.32, CI1.63 to 17.41 ), Grade II vs Grade 0 (OR- 7.5, CI- 1.39 to 40.64)] were significant predictors of mortality. (figure 1)

Conclusions AP patients with ascites have a more severe disease course with a poorer outcome as compared to those who do not have ascites. A higher degree of ascites and IAH grades are significant predictors of mortality.

\section{IDDF2019-ABS-0181 ENDOSCOPIC AND MICROBIOLOGICAL CHARACTERISTICS OF PATIENTS WITH FUNGAL ESOPHAGITIS}

${ }^{1}$ Binh Nguyen Phuc*, ${ }^{2}$ Hang Dao Viet, 'Dung Nguyen Tuan, ${ }^{1}$ Vung Vu Thi, ${ }^{2}$ Linh Nguyen My, ${ }^{2}$ Long Dao Van. 'Institute of Gastroenterology and Hepatology, Vietnam; ${ }^{2}$ Hanoi Medical University, Vietnam

\subsection{6/gutjnl-2019-IDDFabstracts.186}

Background Fungal esophagitis is an uncommon condition which is usually found in patients with an impaired immune system. Patients with normal immune system could also develop fungal esophagitis. In Vietnam, there have not been many researches on fungal esophagitis with details of endoscopic findings and culture results. This study aimed to evaluate the severity of fungal esophagitis by Kodsi classification and identify types of fungi.

Methods An observational study was conducted at the Institute of Gastroenterology and Hepatology from November 2017 to October 2018. Biopsy specimens of patients with suitable endoscopic findings and positive direct microscopic detection were sent to the National Hospital of Dermatology and Venereology for fungal culture and identification. The study collected endoscopic findings, the severity of fungal esophagitis assessed by Kodsi classification and fungal culture results.

Results The prevalence of fungal esophagitis in the total number of upper endoscopies during the study time was $1.4 \%$. 165 patients (86 males and 76 females) were included in the study with the mean age of $51.8 \pm 11.9$ (50-59 years old group was predominant). $44.2 \%$ of patients had other esophageal lesions. The proportions of patients with fungal esophagitis grade I, II and III according to Kodsi classification were $47.3 \%, 47.3 \%$, and 5.4\%, respectively. 155 patients had positive fungal culture results, among them: 143 (92.3\%) were Candida albicans, $11(7.1 \%)$ were other species of Candida, and 1 was Fusarium oxysporum (table 1).

\begin{tabular}{lcc} 
Abstract IDDF2019-ABS-0181 & Table $\mathbf{1}$ & \\
\hline Fungi species & N & $\%$ \\
\hline Candida albicans & 143 & 92.2 \\
Candida & 2 & 1.3 \\
glabrata & & \\
Candida krusei & 3 & 1.9 \\
Candida spp & 6 & 3.9 \\
Fusarium & 1 & 0.7 \\
oxysporum & & \\
\hline
\end{tabular}

Conclusions Fungal infection coexisted with other esophageal lesions. Most patients had mild fungal esophagitis. The proportion of positive fungal culture was high with the predominance of Candida albicans.

\section{IDDF2019-ABS-0186 CORRELATION BETWEEN SEROLOGICAL BIOMARKERS AND DISEASE ACTIVITY IN PATIENTS WITH INFLAMMATORY BOWEL DISEASE}

Mengque Xu*, Xiaoli Chen, Mengsha Cen, Qian Cao. Sir Run Run Shaw Hospital, Zhejiang University, China

\subsection{6/gutjnl-2019-IDDFabstracts. 187}

Background Current biomarkers have been routinely non-invasive methods for assessing disease activity of inflammatory bowel disease (IBD), but none of them are specific. This study was aimed to determine the performance of the serological biomarkers for detecting disease activity in patients with IBD. Methods A prospective study that included 73 ulcerative disease (UC) subjects, 141 Crohn's disease (CD) subjects, and 30 of them were complicated with $C$. difficile infection (CDI), randomly selected from a single-institution IBD database. Disease activity was assessed using by Truelove and Witts criteria for UC and Harvey Bradshaw Simple Index for CD. Serological inflammatory biomarkers were compared in different severity groups. Receiver operator curve analyses assessed the performance of each biomarker in discriminating disease states.

Results For UC patients, elevated monocyte counts, C reactive protein (CRP) and decreased lymphocyte counts, lymphocyte/ monocyte ratio (LMR) significantly differed between subjects with active UC and UC in remission, and LMR performed better than the other leukocyte profiles. LMR of 3.1 was $76 \%$ sensitive and had a specificity of $67 \%$ for active UC (figure 1). CRP and fibrinogen were significantly elevated in UC with CDI compared to inactive UC without CDI patients. For CD patients, higher values of white blood cells, neutrophils, monocytes, neutrophil/lymphocyte ratio, CRP fibrinogen, and lowe values of LMR and hemoglobin were significantly different between subjects with active $\mathrm{CD}$ and $\mathrm{CD}$ in remission. None of the biomarkers included had a good correlation with disease activity (Area Under the ROC curve [AUC] $<0.70$ ) (figure 2). CD with CDI patients compared with CD patients in remission showed significantly higher CRP.

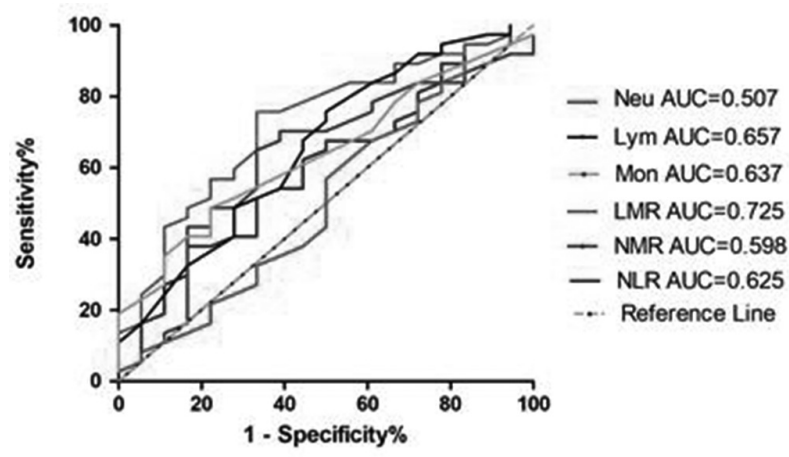

Abstract IDDF2019-ABS-0186 Figure 1 Biomarker performance. Receiver operator curves of the various leukocyte subtypes and ratios in UC active compared with UC remission 